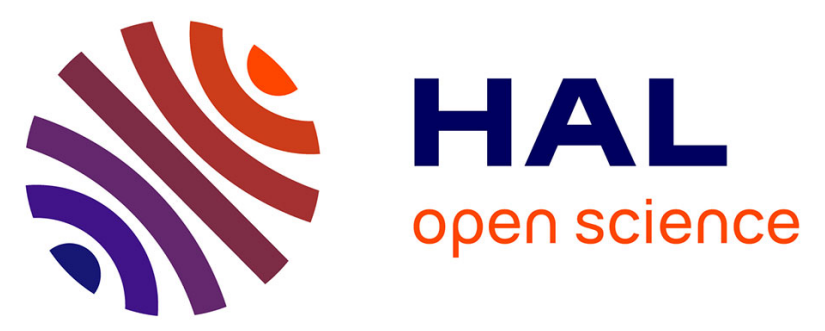

\title{
The Paradigm Shift in Smart Manufacturing System Architecture
}

\author{
Yan Lu, Frank Riddick, Nenad Ivezic
}

\section{To cite this version:}

Yan Lu, Frank Riddick, Nenad Ivezic. The Paradigm Shift in Smart Manufacturing System Architecture. IFIP International Conference on Advances in Production Management Systems (APMS), Sep 2016, Iguassu Falls, Brazil. pp.767-776, 10.1007/978-3-319-51133-7_90 . hal-01615806

\section{HAL Id: hal-01615806 https://hal.inria.fr/hal-01615806}

Submitted on 12 Oct 2017

HAL is a multi-disciplinary open access archive for the deposit and dissemination of scientific research documents, whether they are published or not. The documents may come from teaching and research institutions in France or abroad, or from public or private research centers.
L'archive ouverte pluridisciplinaire HAL, est destinée au dépôt et à la diffusion de documents scientifiques de niveau recherche, publiés ou non, émanant des établissements d'enseignement et de recherche français ou étrangers, des laboratoires publics ou privés. 


\title{
The Paradigm Shift in Smart Manufacturing System Architecture
}

\author{
Yan Lu, Frank Riddick, and Nenad Ivezic \\ Systems Integration Division, NIST, Gaithersburg, USA \\ \{yan.lu, frank.riddick, nenad.ivezic \}enist.gov
}

\begin{abstract}
Smart Manufacturing seeks to integrate advanced manufacturing methods, operational technologies (OT), and information and communication technologies (ICT) to drive the creation of manufacturing systems with greater capabilities in cost control and performance. A crucial differentiation of smart manufacturing systems (SMS) lies in their architectures, which are organized as networks of cooperating manufacturing components specialized for different functions as opposed to the previous organization characterized by rigid, hierarchically-integrated layers of application components. This "ecosystem" of manufacturing components enables SMS that can provide heretofore unattainable levels of performance for manufacturers with respect to agility, productivity, and quality. This paper provides a study of the architectural impact of individual ICT technologies on the emerging manufacturing ecosystem that potentially eliminates the need to design manufacturing systems based on the hierarchical levels of the legacy ISA 95 model. Additionally, we propose a service-oriented SMS architecture that leverages the benefits of ICT and the safety and security requirements from the OT domain. Key challenges of implementing such architectures are also presented.
\end{abstract}

Keywords: System architecture - Smart manufacturing - Cyber physical production system $\cdot$ Service oriented architecture

\section{$1 \quad$ Introduction}

In the current market, customers call for products tailored to their particular needs. Manufacturers seek to meet the demand for these products, but many current manufacturing systems cannot meet the increased requirements for product customization at mass production rates that are profitable. Yet, advanced manufacturing methods, such as 3D printing and flexible production, are emerging with the promise to make highly-personalized production at scales both possible and affordable. Simultaneously, rapid advances in information and communications technologies (ICT) are being applied to manufacturing systems, driving a shift from traditional labor-intensive processes to advanced-technology-based processes 0 . This on-going integration of ad-

adfa, p. 1, 2011.

(C) Springer-Verlag Berlin Heidelberg 2011 
vanced manufacturing methods, operation technologies (OT), and the ICT technologies, is fueling the current Smart Manufacturing (SM) trend to enable systems that can respond in real time to meet changing demands and conditions in the factory, in the supply network, and in customer needs 2 .

Historically, manufacturing systems have been designed to follow the Purdue Reference Model for Computer Integrated Manufacturing 2, which was standardized in ISA954. The ISA 95 model includes 5 levels of manufacturing functions that are often implemented as logically separated layers of applications or systems. Specifically, lower level systems $(0,1$ and 2$)$ and higher levels applications ( 3 and 4$)$ are separated into the $\mathrm{OT}^{1}$ and information technology (IT) domains. The legacy architecture has been widely adopted and implemented in last 30 years accompanied by hierarchical, diverse and domain specific communication structure 7. However, under the legacy architecture, not only is integration between IT and OT difficult, but skip-level function integration is not supported, which makes it too rigid to adapt rapidly to evolving opportunities from ICT technology integration. For example, in the Internet of Things (IoT) world, every part of a manufacturing enterprise is designed to be connected on the internet so that communication, integration and automation can be achieved without constraints. In addition, cloud and mobile computing enable manufacturing functions once implemented at different levels of the hierarchy to now be available without even knowing where they are hosted. Smart components and smarter systems on the shop floor can run advanced analytics and simulations, and make decisions beyond the lower functions defined in ISA 95.

This suggests that a new, information-centric architecture is needed in order to realize ICT's full potential for SMS and facilitate transformation of closed or proprietary manufacturing system architectures into networks of cooperating manufacturing components so as to attain higher degrees of flexibility and integration. This paper provides a study of the architectural impact of ICT on a manufacturing ecosystem and proposes a service oriented architecture that leverages the benefits of ICT and safety and security requirements from the manufacturing OT domain.

The rest of the paper is structured as follows. Section 2 defines a manufacturing ecosystem to scope our research followed by a description of the legacy architectural model based on ISA 95. Section 3 describes the key ICT technologies identified that contribute most to the SMS architecture paradigm shift and presents their architectural impacts on SMS. In Section 4 we propose a new SMS reference architecture. Key challenges of implementing such an architecture are presented. Section 5 concludes the paper with future research direction.

\footnotetext{
${ }^{1}$ Operational technology (OT) is hardware and software that detects or causes a change through the direct monitoring and/or control of physical devices, processes and events in the enterprise. http://www.gartner.com/it-glossary/operational-technology-ot/
} 


\section{SMS and the Legacy Architecture}

Smart manufacturing encompasses a broad scope of systems in a manufacturing business including production, management, design, and engineering functions. The collection of hardware components, their related software components, and the support applications that make up a manufacturing enterprise are what we term the smart manufacturing ecosystem 45 . Figure 1 illustrates three dimensions of concern that are manifest in the smart manufacturing ecosystem. Each dimension - product, production system, and business - is shown within its own lifecycle. Each of these dimensions comes into play in the vertical integration of machines, plants, and enterprise systems in what we call the Manufacturing Pyramid.

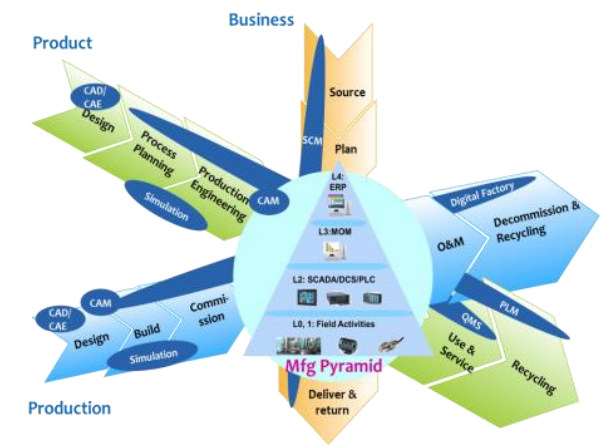

Fig. 1. Manufacturing Ecosystem (Source: 245)

The Manufacturing Pyramid, in the center of Figure 1, is often referred to as the manufacturing system and implemented based on the ISA 95 model. The ISA 95 standard defines a 5-level model for activities in the manufacturing enterprise. Each level provides specialized functions and has characteristic response times 7 . Level 0 and Level 1 define field-level activities including the actual physical production process and the sensing and manipulating of the process. Level 2 defines the functions of monitoring, automated control, and supervisory control of the production process, which usually are performed by programmable logic controllers (PLC), distributed control systems (DCS) and/or supervisory control and data acquisition systems (SCADA). Level 1 and Level 2 communicate within time frames on the order of hours, minutes, seconds, and sub-seconds. Level 3 defines the Manufacturing Operation Management (MoM) activities of work flow and stepping the process through states to produce the desired end products. It deals with maintaining records and optimizing the production process. Level 3 deals with time frames of days, shifts, hours, minutes, and seconds. Level 4 defines the Enterprise Resource Planning (ERP) activities to establish the production (master) schedule (i.e., what to produce, which materials to use, where to deliver the product, and how to ship it there). It deals with determining inventory levels and making sure that materials are delivered on time to the right place for production. Level 4 deals with time frames of months, weeks, days, and shifts. 
While the ISA 95 model is used widely to support the definition of architectures for manufacturing systems, recently some drawbacks with its use have emerged 7 . These drawbacks include:

1) Integrating systems across level boundaries is difficult due in part to the use of different network technologies on different levels.

2) Integrating between Supply Chain Management (SCM), Customer Relation Management (CRM), and Product Lifecycle Management (PLM) activities on different ecosystem dimensions is not supported.

Various methods have attempted to deal with the drawbacks of systems architected based on the ISA95 model. Some software vendors and system integrators have provided solutions focused on the vertical integration of manufacturing components in the manufacturing pyramid to enable advanced controls at the shop floor and optimal decision making at the plant and enterprise. Efforts have also been observed that focus on exchanging information between manufacturing ecosystem dimensions, such as continuous process improvement (CPI) programs, flexible manufacturing systems (FMS), and design for manufacturing and assembly (DFMA) approaches. Some lean enterprise efforts connect SCM with ERP to reduce inventory and enable demand-based manufacturing 5. However, these individual efforts fall short in providing widely applicable methods for integration within and across the three ecosystem dimensions required for SMS. The combination of these perspectives requires a new reference architecture.

\section{Architectural Impacts of ICT Technologies}

For our study, ICT technologies are classified in three clusters: (1) digital technology including digital thread, product/production/process modeling and simulation, knowledge management, and visualization; (2) infrastructure technology including IoT, big data and advanced computing; and (3) Smart systems technology involving Cyber Physical Production System (CPPS) and service-oriented architecture. All technology of the three clusters contribute to the paradigm shift from the legacy architectures to a new SMS architecture. Each cluster is described in the sections below.

\subsection{The Impact of Digitalization}

An enormous amount of information is generated and used during the product, production system and business lifecycles. This data might be used to ensure customer requirements are met, to assess product and process performance, or to meet environmental reporting requirements. Digitalization is the process through which information about the product, processes, and production chain are rendered in a digital form that can be archived, exchanged, or analyzed electronically. Digitalization capabilities help manufacturing companies create virtual representations of their products, assets and processes, exchange large amounts of data rapidly, store data efficiently, enrich processes with digital expert knowledge, generate valuable insights from analyzing "big data", and facilitate communication and collaboration through digital channels within their value chain 9. 
The pervasive adoption of digital technology will have two dominant impacts on SMS and their architecture.

1) There will be an increase in the availability of collaborative environments for product development. Their architectures will exhibit a tighter integration of PLM, ERP, MES, SCM, CRM and asset management functions. These platforms will enable customers and suppliers to directly participate in product design and manufacturing. In this way, high-quality customized products can be manufactured and increased traceability can be achieved. Direct feedback from manufacturing and product use to product development will shorten product innovation cycles.

2) Digital representations of products, physical assets, production operations, and business processes will enable the creation of a digital "twin" of the factory-a comprehensive virtual representation of a real factory and its processes that can be made to run in parallel to the real factory20. Models and simulations can be instantiated with product and production system data and used in the real factory decision making processes to optimize business, manufacturing, and supply chain operations and to improve product development processes. At the same time, the data coming from the real factory can be fed back to the digital factory to improve the models, knowledge bases, and simulations.

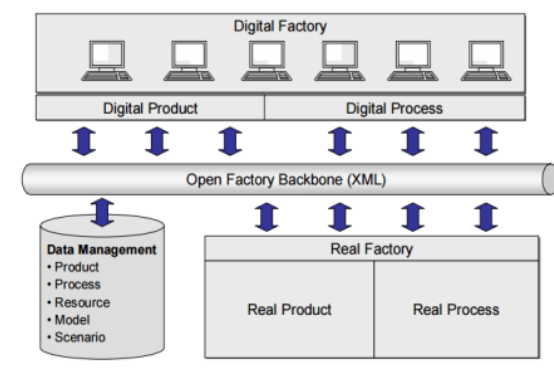

Fig. 2. Digital twin for manufacturing (Source: 9)

\subsection{The Impact of IoT}

The development and adoption of the Internet of Things (IoT) is a critical element of Smart Manufacturing 10. The technologies that will become pervasive because of the IoT-network-addressable physical and application components and standard communication protocols through which those components can communicate-will enable enterprise architectures, in general, and industrial system architectures, in particular, to move on from their traditional hierarchically and domain-specific network structure to a unified and IP-based structure10, as shown in Figure 3. This shift will enable enterprise services, such as data analytics and edge computing services, that today are typically only available at the highest levels of an enterprise to be available to serve system components in all areas of the industrial enterprise. 


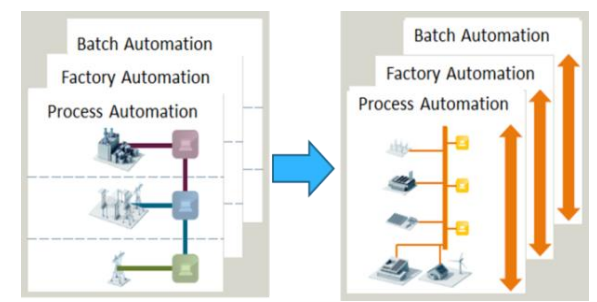

Fig. 3. Ubiquitously connected manufacturing systems (Source: adapted from 10)

The transition to more open network architectures, combined with Big Data and Cloud computing, will bring profound opportunities to SMS. At the same time, new security challenges are presented with billions of new smart devices being interconnected in the world of the IoT 11. The biggest security concern comes from connecting IoT devices, sensors, actuators and edge-computing units, with existing controllers, automation and manufacturing information networks, and applications. Existing OT and IT security approaches and policies will need to be adapted to embrace these new IoT security challenges. Most automation and information suppliers have established industry security services to assist clients to assess security risks and harden their manufacturing plants. Reference network architectures are available from both network equipment and automation system vendors 12. Additional challenges of integrating IOT include unreliable communications of sensors and actuators on IP networks, how to leverage IoT to boost computing power for advanced manufacturing analytics, and accessibility issues of gaining accesses to the legacy manufacturing assets.

\subsection{The Impact of CPPS}

While the IoT deals with unique, identifiable, and internet-connected physical objects, cyber-physical systems efforts are concerned with the nature of cyber-physical coupling and the system of systems characteristics of networked and software-controlled systems. CPPS is considered the core component for German Industrie 4.0 program, where they are also referred to as I4.0 Components 13. SMS are essentially composed of CPPS, which can respond intelligently to changing tasks and conditions and reconfigure themselves. CPPSs partly break the traditional automation pyramid and potentially turn the manufacturing ecosystem into a service-oriented architecture (SOA) 1516. To leverage the safety and security requirements from the OT domain, real-time and safety critical functions in legacy automation systems will remain organized in a hierarchy, and only level 2 components will be allowed to connect as services. IoT devices connected in the IT world will naturally be part of the service-oriented architecture. Figure 4 shows how the legacy hierarchical functional architecture will be transformed to a SOA-based manufacturing system architecture. 


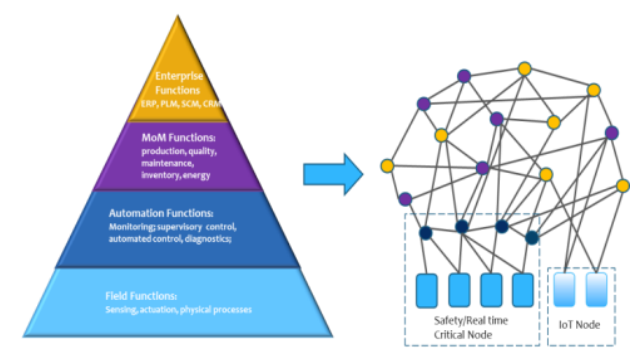

Fig. 4. CPPS Impact on manufacturing system function architecture (Source: Adopted from 18)

\section{Smart Manufacturing Architecture}

With the architectural impacts from ICT technologies in mind, we propose a SOA for Smart Manufacturing systems.

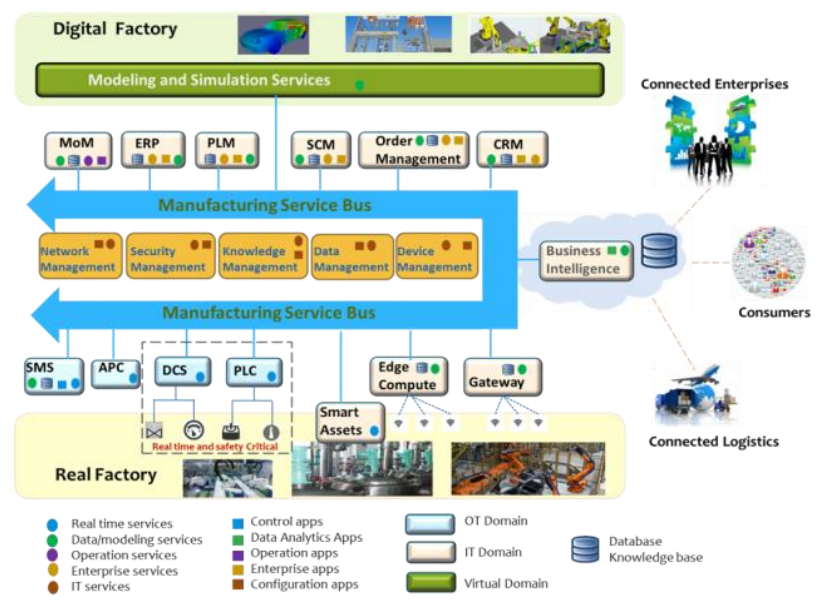

Fig. 5. A Service Oriented and Model based SMS Architecture

As shown in Figure 5, the proposed architecture provides a completely integrated manufacturing ecosystem on a single manufacturing service bus, including both OT domain systems and IT domain systems and applications. Interactions with suppliers, customers, and logistics systems are enabled through a collaborative Business intelligence (BI) service.

In the new architecture, services are classified as being one of four types: IT domain services, OT domain services, virtual domain services and management/common services. IT domain services include IoT services (e.g., smart devices, edge computing, fog computing, cloud computing, etc.), MoM services, ERP services, PLM services, and other enterprise services such as Asset Management, Energy Management, SCM, Order Management and CRM. The integration approach that will be used for IT domain services is similar to today's enterprise service bus (ESB) based approach, which is 
built using event-driven and standards-based message-oriented middleware in combination with message queues. In the OT domain, services involve functions provided by machines, workstations, robots, production lines, complete production cells or even products themselves. The OPC Unified Architecture (OPC UA) provides interoperability at level 2 and beyond and has the potential to provide a service-oriented architecture for OT application integration13. Data analytics services are provided through edge computing, fog computing and cloud computing in the IoT world 18. Virtual domain services are located in the digital factory, which stores the data models, function models, behavior models, and process models of a manufacturing enterprise and provides query and simulation functions for manufacturing applications. Management/common services sit at the core of the SOA architecture. They help manage the lifecycle of the SMS services, map client requests with service providers, and ensure quality of service levels met. Device management, network configuration, data management, knowledge management, and security management are provided by services in this cluster.

Based on the architecture described above, SMS can be developed and deployed that provide efficient and flexible solutions for the problems faced by the modern manufacturing enterprise. Supporting more streamlined processes, the SOA-based architecture enables customized production at mass production rates.

However, implementing the proposed architecture to create new SMS is neither simple, quick, nor cheap. It will take many years to fully realize the strength of the new architecture. The key implementation challenges include:

- Manufacturing Service Bus capabilities - can a single manufacturing service bus meet the needs of diverse use scenarios, supporting both real-time messaging and big volume data distribution?

- Real-time service capabilities - how should real-time services be modeled in the OT domain, how should their service interfaces be defined, and how should resource constraints be managed, i.e., when and where should resources be available to provide services?

- IT - OT integration concerns - how can security and safety issues be handled?

- Can high fidelity models and simulations be used in a real-time control environment without re-engineering?

- Knowledge management concerns - how can we close technology gaps in conditioning, interpreting, and contextualizing data across heterogeneous systems or situations?

- Integration standards - how can existing standards be enhanced and new standards be developed to enable more information flow among more stakeholders.

\section{Conclusion}

In a new era of smart manufacturing, every part of a manufacturing enterprise will be digitized and connected. With the introduction of smart devices accessible as services on a network, more embedded intelligence at every level, predictive analytics, and cloud technology, the next generation of manufacturing systems will indeed get smarter. In order to realize the vision of smart manufacturing where systems respond 
in real time to changing demands and conditions in the factory, in the supply network, and in customer needs, the classical manufacturing system architectural paradigm based on a hierarchical control model will no longer dominate. A new paradigm based on distributed manufacturing services is starting to be adopted.

This paper described a smart manufacturing ecosystem that enabled systems integration within and across three manufacturing lifecycle dimensions - product lifecycle, production system lifecycle, and business lifecycle. We examined how the introduction of ICT is going to impact the SMS architecture. Adoption of IoT, CPPS and cloud computing technology potentially will drive the transformation of the existing rigid hierarchical architecture style. Digital thread and digital factory technology will enable the enterprise to be fully integrated with its value chain. Based on the study of the ICT architectural impact, we propose an SOA-based and model-enabled Smart Manufacturing reference architecture. The proposed reference architecture will integrate both ICT and OT systems on a single manufacturing service bus. We discussed implementation challenges that include standards for communication protocols, data model, knowledge representation, and CPPS characterization that are necessary to facilitate the adoption of Smart Manufacturing technology and the proposed architecture.

Disclaimer. Any mention of commercial products is for information only; it does not imply recommendation or endorsement by NIST.

\section{References}

1. Institute for Defense Analyses, https://www.wilsoncenter.org/sites/default/files/Emerging_Global_Trends_in_Advanced_Manufacturing.pdf

2. Frechette, S., Morris, K.C., Lu, Y: Smart Manufacturing Isn't So Smart Without Standards, http://blog.mesa.org/2016/03/smart-manufacturing-isnt-so-smart.html

3. Williams, T. J.: The Purdue Enterprise Reference Architecture: A Technical Guide for CIM Planning and Implementation, Instrument Society of America, 1 (1992)

4. ANSI/ISA-95.00.01-2010 (IEC 62264-1 Mod): Enterprise-Control System Integration - Part 1: Models and Terminology (2010)

5. Lu, Y., Morris, K.C., Frechette, S. P.: Standards Landscape and Directions for Smart Manufacturing Systems, IEEE Conference on Automation Science and Engineering, Gothensburg (2015)

6. Lu, Y., Morris, K.C., Frechette, S. P.: Current Standards Landscape for Smart Manufacturing Systems, NISTIR 8107 (2016)

7. Brandl, D.: The IT Implications of ISA 95 and ISA 99, http://www.brlconsulting.com/Files/The\%20IT\%20Implications\%20S95\%20and\%20S99.pdf

8. LNS Research: Agile MES and IIoT: How the Traditional Hierarchies in Manufacturing Operations Management Are Being Dissolved, Webinar, Feb. 29 (2016)

9. Wolfgang, K.: Digital Factory-Integration of Simulation Enhancing the Product and Production Process Towards Operative Control and Optimisation. International Journal of Simulation: Systems, Science and Technology, 7(7) (2006)

10. Lopez Research: https://www.iotwf.com/resources/6 
11. Guido, S. Internet of Thing and Services, Siemens Future Forum@ Hanover Messe 2014, https://w3.siemens.com/topics/global/en/events/hannover-messe/program/Documents/pdf/Internet-of-Things-and-Services-Guido-Stephan.pdf

12. Davidson, M.: IoT in Manufacturing Hurdle \#2: New Security Challenges, July, 2014, http://blog.lnsresearch.com/blog/bid/199506/IoT-in-Manufacturing-Hurdle-2-New-Security-Challenges

13. GE's Intelligent Platforms business \& CISCO, http://www.cisco.com/c/dam/en_us/solutions/industries/docs/manufacturing/architecting-robust.pdf

14. VDI/VDE Society Measurement and Automatic Control (GMA), Reference Architecture Model Industrie 4.0 (RAMI4.0) (2015)

15. Boyd, Alan, D. Noller, P. Peters, D. Salkeld, T. Thomasma, C. Gifford, S. Pike, and A. Smith.: SOA in Manufacturing-guidebook, MESA International, IBM Corporation and Capgemini Co-Branded White Paper (2008)

16. IBM: SOA Approach to Enterprise Integration for Product Lifecycle Management. IBM, International Technical Support Organization (2008)

17. Monostori, L.: Cyber-physical Production Systems: Roots, Expectations and R\&D Challenges, CIRP 12/2014, 17, 9-13 (2014)

18. Abdelshkour, M.: IoT, from Cloud to Fog Computing, http://blogs.cisco.com/perspectives/iot-from-cloud-to-fog-computing

19. Vogel-Heuser, G., Kegel, G., Bender, K., Wucherer, K.: Global Information Architecture for Industrial Automation. Automatisierungstechnische Praxis (atp), Oldenbourg-Verlag, Muenchen (2009)

20. Michael Grieves, http://innovate.fit.edu/plm/documents/doc_mgr/912/1411.0_Digital_Twin_White_Paper_Dr_Grieves.pdf 